\title{
The Minimum Entropy Production Principle and Heat Transport in Solids with Internal Structure ${ }^{\dagger}$
}

\author{
Ruth Estephania Gonzalez Narvaez ${ }^{1}$, Federico Vazquez Hurtado ${ }^{2}$ and Mariano López de Haro ${ }^{3}$ \\ 1 UAEM, Toluca, Mexico \\ 2 CiNC UAEM, Cuernavaca, Mexico \\ 3 IER UNAM, Temixco, Mexico \\ + Presented at the Entropy 2021: The Scientific Tool of the 21st Century, 5-7 May 2021; Available online: \\ https://sciforum.net/conference/Entropy2021/.
}

Published: 5 May 2021

Variational principles have a long story in the study of the time evolution of dissipative systems. There is a wide variety of formulations of such principles, some of which are ad hoc techniques like doubling of the dynamic variables, restricted variations, etc. There also exist some principles which have a fundamental character like the minimum entropy production principle, MEPP, which refers to the stationary state eventually reached by a system after it has been taken out of equilibrium. Much has been discussed whether the MEPP has general application or whether it is of rather limited validity. In particular, it has been concluded that for systems with constant phenomenological Onsager coefficients the entropy production can only decrease in time until a minimum is reached when the system is in the stationary state, it yet being on debate. In this work heat transport in non-homogeneous solids is considered. We study the case of solids with internal structure within the framework of a two temperature description. The internal structure is introduced in the model through the dependence of the thermal conductivity on position. The time evolution equations are obtained through the usual methods of irreversible thermodynamics and from the MEPP. We find that in our approach both sets of evolution equations coincide and that, without imposing any restriction on the phenomenological coefficients other than those coming from the internal structuring of the solid, the appropriate temperature profiles are obtained. We exemplify this finding with the case of pure Aluminum subjected to a heat pulse.

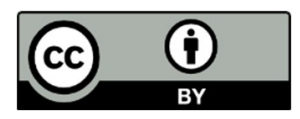

(C) 2021 by the authors. Licensee MDPI, Basel, Switzerland. This article is an open access article distributed under the terms and conditions of the Creative Commons Attribution (CC BY) license (http://creativecommons.org/licenses/by/4.0/). 